This is an author produced version of a paper published in Agile Processes, in Software Engineering, and Extreme Programming. This paper has been peer-reviewed but does not include the final publisher proof-corrections or journal pagination.

Citation for the published paper:

Berger, Christian; Eklund, Ulrik. (2015). Expectations and Challenges from Scaling Agile in Mechatronics-Driven Companies : A Comparative Case Study. Agile Processes, in Software Engineering, and Extreme Programming, p. null

URL: https://doi.org/10.1007/978-3-319-18612-2_2

Publisher: Springer

This document has been downloaded from MUEP (https://muep.mah.se) / DIVA (https://mau.diva-portal.org). 


\title{
Expectations and Challenges from Scaling Agile in Mechatronics-Driven Companies - A Comparative Case Study
}

\author{
Christian Berger ${ }^{1}$ and Ulrik Eklund ${ }^{2}$ \\ 1 University of Gothenburg, Sweden \\ Department of Computer Science and Engineering \\ christian.berger@gu.se \\ 2 Malmö University, Sweden \\ Department of Computer Science \\ ulrik.eklund@mah.se
}

\begin{abstract}
Agile software development is increasingly adopted by companies evolving and maintaining software products to support better planning and tracking the realization of user stories and features. While convincing success stories help to further spread the adoption of Agile, mechatronics-driven companies need guidance to implement Agile for non-software teams. In this comparative case study of three companies from the Nordic region, we systematically investigate expectations and challenges from scaling Agile in organizations dealing with mechatronics development by conducting on-site workshops and surveys. Our findings show that all companies have already successfully implemented Agile in their software teams. The expected main benefit of successfully scaling agile development is a faster time-to-market product development; however, the two main challenges are: (a) An inflexible test environment that inhibits fast feedback to changed or added features, and (b) the existing organizational structure including the company's mind-set that needs to be opened-up for agile principles.
\end{abstract}

Key words: scaling agile, agile, software development process, mechatronics, comparative case study

\section{Introduction}

Developing high-quality software products that better match a customer's expectations is successfully supported by Agile [1]. Key advantages over other development approaches are short and fixed periods consisting of development, integration, and testing, small team sizes, and active communication within the software team while also including the customer. A flexible development approach allows a team to get frequent feedback to newly added features from the end-user but also enables reprioritization of user stories and feature requests whenever the stakeholders' needs change over time. 
The typical habitat for adopting Agile are pure software-driven companies with prominent examples being Google and Amazon. Implementing Agile in environments where the final product combines software, hardware, and mechanics is more challenging considering the different nature of the involved artifacts.

\subsection{Problem Domain and Motivation}

In the mechatronics domain there are two opposing trends affecting R\&D: Manufacturing and hardware development is a mature domain, which has been optimized for more than fifty years, but still having long lead-times, typically years. Focus during $R \& D$ is on predictability, i.e. meeting the start-of-production (SOP) with the required mechanical quality, which in practice is achieved by stage-gate/waterfall processes. In contrast, software development today is characterized by increasing speed and being more nimble while keeping quality. This typically enables lead-times of weeks or months, and many agile methods are a response to this. There are no established solutions to solve the intersection between the aforementioned trends, but the necessity to resolve them in the mechatronics domain motivates further studies.

\subsection{Research Goal}

The goal for this comparative study is to systematically investigate expectations and challenges from scaling Agile outside software teams on the example of three companies from the Nordic region developing and manufacturing embedded and mechatronic products. Specifically, we are interested in the following subgoals:

1. Unveiling expectations and challenges originating between teams, departments, and divisions,

2. Unveiling challenges from mechatronics-related development-, project-, and product-processes, and

3. Understanding expectations from key stakeholders like teams, managers, and organizations at large.

\subsection{Contributions and Scope}

We designed and conducted a comparative case study at three companies and report about our findings according to Runeson and Höst (cf. [2]). The main contributions of this work are:

1. Defining a methodology to systematically unveil and compare expectations and challenges for scaling Agile in mechatronics-driven organizations,

2. Presenting results from individual on-site workshops at the three different mechatronics companies, and

3. Summarizing results from a joint follow-up survey at all companies based on the results from the individual workshops. 


\subsection{Structure of the Article}

The rest of the article is structured as follows: Section 2 presents related work in this field. Section 3 describes the design of the comparative case study and the embodied methods followed by the results from the comparative case study in Section 4. Section 5 presents conclusions from our study.

\section{Related Work}

Originally, agile methods evolved to meet the needs of small and co-located development teams [3]. They typically emphasize close customer collaboration, iterative development, and small cross-functional development teams. Also, team autonomy and end-to-end responsibility are reported as important characteristics permeating the methods [4]. Most companies introduce agile methods to increase the frequency in which they release new features and new products, and as a way to improve their software engineering efficiency. According to Dingsøyr et al. [5], agility embraces lean processes with an emphasis on realizing effective outcomes, and common for agile methods is that they entail the ability to rapidly and flexibly create and respond to change in the business and technical domains [5].

Due to many successful accounts [6, 7], agile methods have become attractive also to companies involved in large-scale development of embedded systems, and several attempts to extend agile methods to include development of embedded systems are seen $[8,9,10]$.

While convincing success stories from industry help to further spread the adoption of Agile, there are few studies of agile development focusing on the mechatronics domain. There are examples of some companies successfully introducing agile practices at the team level, typically characterized by individual teams defining their own ways-of-working to facilitate speed, short iterations, and delivery quality when developing their components. The experiences thereof are generally positive according to two literature reviews by [11] and [12]. There are also some publications stating that a third of German and American automotive development teams using agile practices reported in a commercial survey [13]. However, with characteristics such as hardware-software interdependencies, heavy compliance to standards and regulations, and limited flexibility due to real-time functionality [14], the development of embedded and mechatronic systems seems to challenge common practices of agile development.

\section{Comparative Case Study Design}

We addressed the aforementioned research goal by designing a comparative case study, where we collected data from three different mechatronics-driven companies. 


\subsection{Research Questions}

We derived the following research questions for the comparative case study:

$R Q-1$ : Which practices from Agile are in use in a mechatronics-driven organization?

$R Q$-2: How is the current implementation of Agile perceived in a mechatronicsdriven organization?

RQ-3: What are the expectations from scaling Agile within a mechatronics-driven organization?

$R Q$-4: What are the main foreseeable challenges when scaling Agile in mechatronicsdriven organizations to achieve the expected benefits?

\subsection{Case and Subjects Selection}

We conducted our research in the context of the Software Center ${ }^{1}$. The Software Center is a cooperation environment where different companies from the Nordic region collaborate with selected universities on research topics and technology transfer from academia to industry. The participating companies in the Software Center cover domains like Automotive, Telecommunication, Mobile Phones, and Defense.

For our comparative case study, we selected three large companies who are mainly mechatronics-driven in their business to which we are referring to as company A, B, and C. The companies employ between approximately 18,000 and 93,000 people and their respective yearly manufacturing of mechatronic products ranges from 0.4 to over 16 million units according to their respective annual reports from 2013. These companies can be considered to be representative due to their individual market shares. Furthermore, all companies have already adopted Agile at team-level in their R\&D departments and apply it since several years during the software development of projects with varying sizes. For the workshops and surveys, participants covered experienced developers and managers from software development, hardware development, integration, and testing.

\subsection{Data Collection Procedure}

The data collection was conducted threefold: (a) We planned and conducted individual on-site workshops at the respective companies in the first phase; (b) the collected data from these individual workshops was analyzed to design a joint survey that was subsequently distributed to key stakeholders within the respective companies in a second phase to enlarge the population for data collection; (c) the feedback from the survey was used to plan and conduct a joint workshop with key representatives from all three companies in the third phase involving an external expert on Agile practices to follow-up on selected key challenges for scaling Agile and to identify topics where to proceed internally at the companies.

\footnotetext{
${ }^{1}$ http://www. software-center.se
} 
Individual On-Site Workshops. The individual workshops were conducted separately for each company. The respective workshop's duration was approximately 3 hours and was moderated by one researcher while the other researcher took notes during the discussion phases. The workshop addressed in a qualitative manner the following two main questions:

1. What would be the biggest benefits if your company successfully scales Agile?

2. What are the challenges for your organization to achieve these benefits?

The participants from different teams (software development, hardware development, and testing) had approximately 20min to write their answers on two-colored sticky notes. The notes were subsequently collected, presented to the audience by the workshop moderator, and clustered during a joint discussion about the respective matter. The resulting topic maps were summarized to identify the key topics for the two aforementioned questions.

Survey. Afterwards, we designed a survey based on the results from three individual on-site workshops according to the guidelines by from Singer et al. published in Shull et al. [15]. The survey was realized as an online questionnaire to reach out to more participants who could not join the on-site workshops ${ }^{2}$. The questionnaire consisted of the following five sections:

1. General data about the role of the participant in the company

2. Use of Agile practices in the company

3. Evaluating the use of Agile in the company

4. Expectations from scaling Agile outside the software development teams

5. Expectations about challenges to be solved when scaling Agile

The first section contained three open-ended questions; the second section contained eight questions to be ranked as Yes, No, and Not applicable and an optional open-ended text field; the third section consisted of eight pairs that needed to be weighted on a scale from 1 to 7 , where 1 means that the entire focus is on the left aspect of the pair and 7 that the entire focus is on the right aspect of the pair; additionally, an optional comment field was available. The fourth section consisted out of 16 expectations for benefits to be ranked on the 6-Likert-scale very important, important moderately important, of little importance, unimportant, and not relevant; this section was complemented with two optional questions asking for further benefits and drawbacks when scaling Agile. The last section consisted of 21 potential challenges collected during the workshops to be ranked on the same 6-Likert-scale as before; this section was also complemented with an optional question asking for further challenges.

The questionnaire was piloted with the single-points-of-contact (SPoC) from the involved companies to improve its logical structure and the overall understanding. The target group for this study contains the attendees of the on-site workshops

\footnotetext{
${ }^{2}$ The survey can be found as supplementary material here: http://goo.gl/yJNez1
} 
extended in snowball manner (cf. Goodman [16]) by the SPoCs to reach out to more employees who are affected when scaling Agile.

Joint Workshop. After conducting on-site workshops and the survey, we organized a joint workshop where we invited delegates from all companies. These delegates covered different departments not only focusing on software development. The goal for the workshop was to present the findings from the separate workshops and the survey, to jointly discuss and complement with missing challenges, and to identify first steps towards initiating initiatives for scaling Agile outside software development teams. For the workshop, we invited an external Agile expert as moderator so that we could follow the discussions among the participants from an observer perspective according to the guidelines from Seaman as published in Shull et al. [15].

\subsection{Analysis Procedure}

Individual On-Site Workshops. Notes were taken during the separate on-site workshops alongside with capturing the resulting topic maps. The notes were structured and summarized as separate reports that were sent to the SPoCs afterwards. The collected clustered topics as well as key statements served as basis for designing the survey.

Survey. The survey was realized as online questionnaire that allowed postprocessing of the data in the statistical environment $\mathrm{R}$. The data was split according to the different sections in the survey and open-ended responses were separated. Likert-visualization was chosen for the range-, pair-focusing, and Likert-scale answers; for the pair-focusing answers, Fisher's exact test (cf. [17]) was chosen to test for differences pairwisely between all companies as this test is robust and applicable even to smaller data sets.

Joint Workshop with External Agile Expert. During the joint workshop, notes were also taken to complement and structure the existing data. The main results from the joined workshop were summarized and sent to attendees afterwards.

\subsection{Validity Procedure.}

To ensure validity in our comparative case study, we applied both, method and data triangulation: For the former, (a) we initially conducted individual on-site workshops to explore the topic at the three different sites, followed by (b) separate surveys at the respective companies with a broad set of recipients, and complemented by (c) a joint workshop from the observer perspective, where we presented results from the first two steps. For the joint workshop, (a) we collected input from different, independent companies, and (b) let the final workshop be moderated by an external person to avoid influencing the workshop outcome. 


\section{Results}

In the following, we are presenting the joint results from the three aforementioned data sources. As the notes from the individual on-site workshops were used to design and structure the survey, they are not reported here explicitly. The survey was completed by 11 respondents from company A, 19 respondents from company $\mathrm{B}$, and 16 respondents from company $\mathrm{C}$ resulting in 46 responses in total.

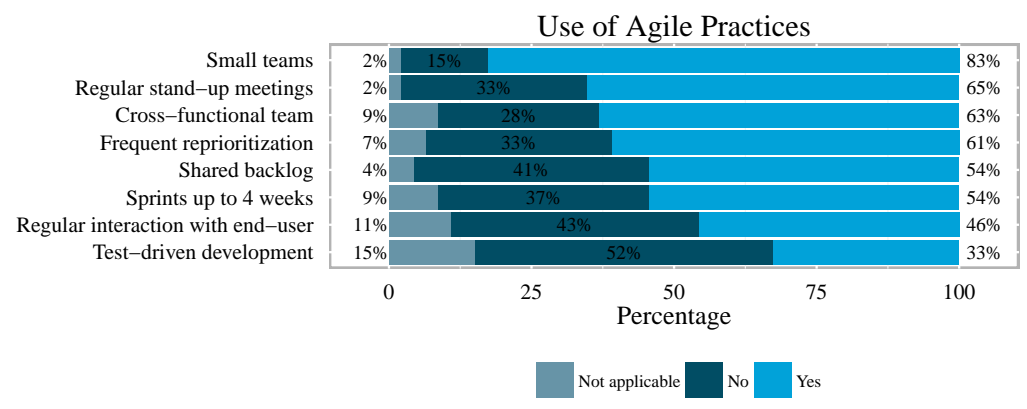

Fig. 1. Familiarity and usage of agile principles over all companies.

Results to RQ-1: Fig. 1 depicts the familiarity and usage of agile principles over all companies. While having small teams is apparently present to a large extent, test-driven development is only applied at one third of the respondents.

Results to RQ-2: The survey's next section asked to estimate where their own company puts its emphasis regarding pairs from opposite aspects regarding agile and non-agile values. Fig. 2 visualizes the responses.

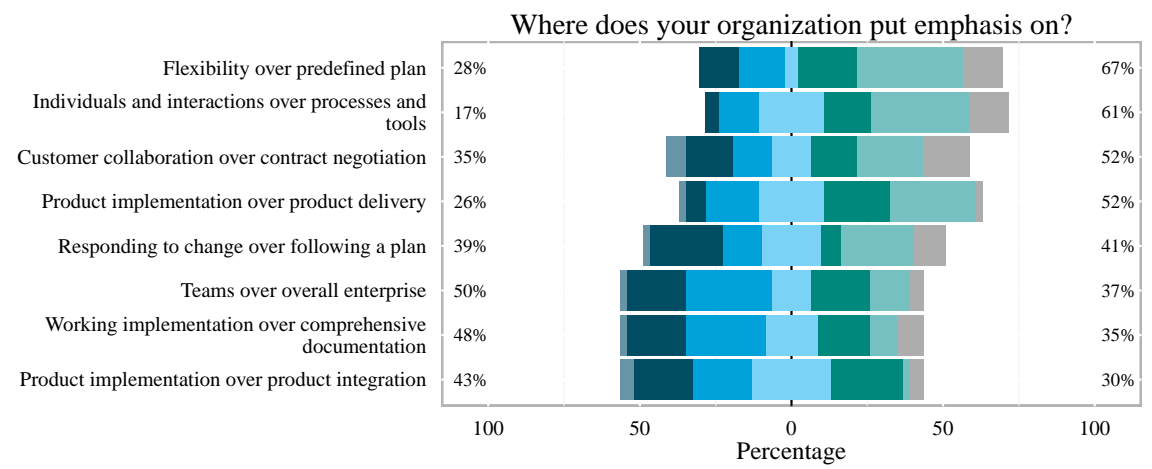

Fig. 2. Where do companies put their emphasis on? Respondents could express their emphasis on a scale from 1 to 7 to describe their level of favoring one topic over the other. 
We conducted a test to pairwisely compare the companies as shown in Tab. 1, and we could not observe any pairwise difference in the responses from the three different companies.

\begin{tabular}{|c|c|c|c|}
\hline \multirow[t]{2}{*}{ Where does your organization put emphasis on? } & \multicolumn{3}{|c|}{ Companies } \\
\hline & $\mathrm{A} / \mathrm{B}$ & $\mathrm{A} / \mathrm{C}$ & $\mathrm{B} / \mathrm{C}$ \\
\hline Individuals and interactions over processes and tools & $p=0.691$ & $p=0.077$ & $p=0.072$ \\
\hline Working implementation over comprehensive documentation & $p=1.000$ & $p=0.400$ & $p=0.272$ \\
\hline Customer collaboration over contract negotiation & $p=0.433$ & $p=0.192$ & $p=0.694$ \\
\hline Responding to change over following a plan & $p=1.000$ & $p=0.666$ & $p=0.476$ \\
\hline Product implementation over product delivery & $p=0.380$ & $p=1.000$ & $p=0.440$ \\
\hline Product implementation over product integration & $p=0.354$ & $p=0.642$ & $p=0.054$ \\
\hline Flexibility over predefined plan & $p=0.679$ & $p=1.000$ & $p=0.452$ \\
\hline Teams over overall enterprise & $p=0.411$ & $p=1.000$ & $p=0.710$ \\
\hline
\end{tabular}

Table 1. Fisher's exact test with a p-value of 0.05 : There is no difference in perceiving a company's emphasis between the responses from pairwisely comparing the companies.

Results to RQ-3: The expected benefits when scaling Agile are presented in the following. As shown in Fig. 3, all companies expect with almost $90 \%$ a higher quality of the work products.

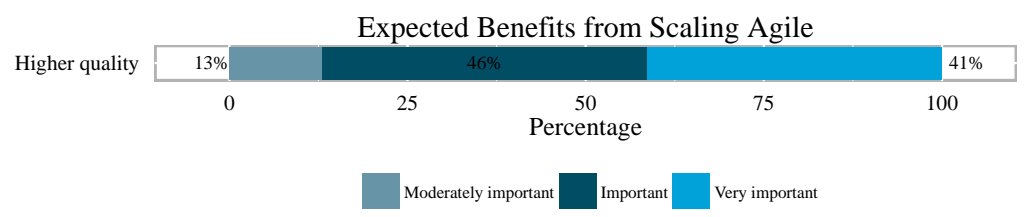

Fig. 3. Higher quality is expected from all companies.

Fig. 4 depicts further expected benefits when scaling Agile where the top responses expect faster time-to-market and shorter lead-times during the development. 


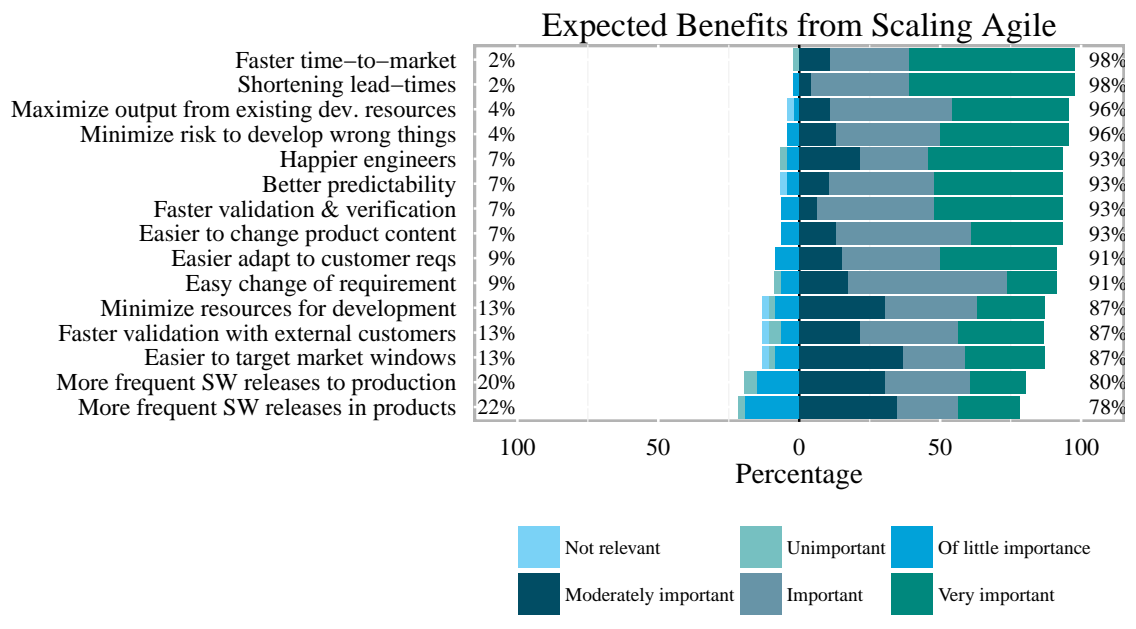

Fig. 4. Expected benefits from scaling agile over all companies.

Results to RQ-4: The expected challenges when scaling Agile are depicted in Fig. 5. The most difficulties are expected in the existing test facilities, which is in line with the low adaptation rate for test-driven development, followed by adapting the organizational structure.

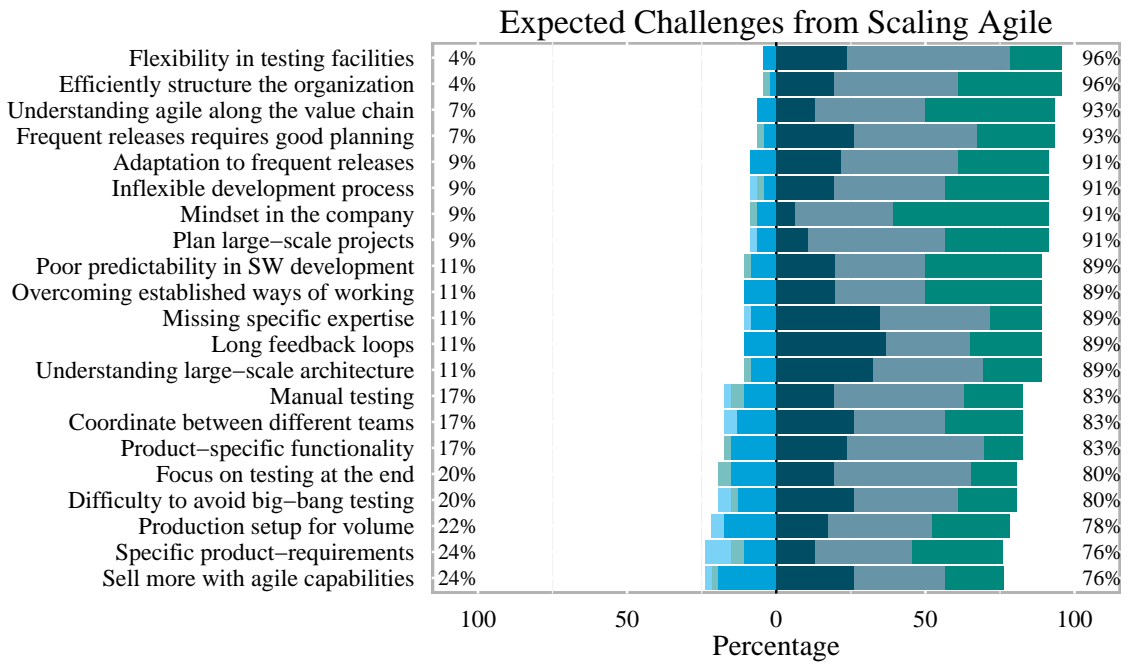

Fig. 5. Expected challenges when scaling Agile over all companies. 
The joint workshop with the external expert on Agile resulted after a discussion phase among the involved companies in the following four cluster areas for expected challenges when scaling Agile: Leadership, Collaboration, Focusing on System, and Focusing on Customer. From these four topic areas where different possible change initiatives were jointly identified, there was consensus between all companies for (a) improving collaboration between all disciplines involved in product development and (b) changing the overall mindset in the organization as initial steps towards scaling Agile outside software development teams.

\subsection{Threats to Validity}

In the following, we are discussing threats to the validity of our comparative case study. Considering construct validity, our method triangulation reduced the risk of capturing incomplete data that would render in misleading results; in this regard, the plausibility of the findings from the different stages was validated with the SPoCs and the final joint workshop. A possible threat to the construct validity is that the survey was based on the underlying assumption that scaled agile development would actually have benefits for the organization, and that assumption may not be shared by respondents to the survey. Furthermore, the authors had only limited influence on the selection of the participants for the workshops.

Regarding internal validity, responses to the expected benefits from scaling Agile were gathered without associating implementation costs to them and thus, enforcing a prioritization. Thus, there might be a tendency from the respondents to wish or hope for all benefits from scaling Agile. As for initial initiatives to scale Agile, the most important challenges are of main interest, this risk, though, can be neglected.

Considering external validity, the selected companies reflect large scale enterprises with more than 15,000 employees and a volume-oriented production process. Furthermore, these companies are leading in their respective market segments and thus, the findings can be generalized to other companies in the mechatronics domains that have a lengthy and traditionally non-agile development process; this observation is also supported by the results from Fisher's exact test.

With respect to reliability, the iterative feedback of the company's SPoCs as well as the involvement of an external expert for Agile, the risk that the findings depend on the involved researchers was tackled.

\section{Conclusion and Future Work}

We presented a comparative case study conducted at three large-scale, mechatronics-driven enterprises to explore benefits and challenges from scaling Agile to non-software teams. The study consisted of individual on-site workshops, a large survey, and a joint workshop with all companies moderated by an external expert on Agile. While all companies have implemented elements from Agile, main findings are that (a) the expected main benefit is a faster time-to-market product development, (b) an inflexible test environment, though, inhibits fast 
feedback to changed or added features and thus, prevents scaling Agile outside the software development team, and (c) the existing organizational structure including the company's mind-set needs to be adapted to beneficially scale Agile.

Relation to Existing Evidence. Our results of the need for an agile mindset and the importance of the testing environment in mechatronics systems is confirmed by other studies. [18] concludes that observed resistance towards working agile was partially based on a lack of an agile mindset, caused by extensive experience with non-agile methods, something also common among the companies in our study. [19] also identified the challenge of realizing continuos integration testing with a wide variety of platforms. One example they mention is the difficulty to reproduce reported faults with the right testing environment including released hardware.

The other main challenge on adjusting the organizational structure confirms what many scaled methods aim for, and is also the topic of both recent research (e.g. $[20,18])$ and of industrial frameworks such as Disciplined Agile Delivery [21].

Impact/Implications. This comparative case study is the first of its kind reporting about explorative results regarding expected benefits and challenges from scaling Agile at large scale, mechatronics-driven companies. Its findings have an apparent impact to companies with a similar development and manufacturing structure.

Limitations. All involved companies are at an comparable stage regarding scaling Agile. Thus, this comparative case study focuses primarily on the expected benefits and the foreseeable challenges when initiating initiatives for scaling Agile outside the software development teams.

Future Work. Future work needs to be done in continuously accompanying the enterprises during their initiatives for scaling Agile to collect and analyze more data towards guidelines and best practices for adopting and scaling Agile in mechatronics companies. Furthermore, comparisons with other domains would be possible to plan and guide such initiatives.

Acknowledgments. We are grateful to the companies who significantly supported this study in the context of Software Center.

\section{References}

1. Beck, K., Beedle, M., van Bennekum, A., Cockburn, A., Cunningham, W., Fowler, M., Grenning, J., Highsmith, J., Hunt, A., Jeffries, R., Kern, J., Marick, B., Martin, R.C., Mellor, S., Schwaber, K., Sutherland, J., Thomas, D.: Manifesto for the Agile Software Development (2001)

2. Runeson, P., Höst, M.: Guidelines for conducting and reporting case study research in software engineering. Empirical Software Engineering 14(2) (2008) 131-164

3. Kettunen, P., Laanti, M.: Combining agile software projects and large-scale organizational agility. Software Process: Improvement and Practice 13(2) (March 2008) $183-193$ 
4. Dybå, T., Dingsøyr, T.: Empirical studies of agile software development: A systematic review. Information and Software Technology 50(9-10) (August 2008) 833-859

5. Dingsøyr, T., Nerur, S., Balijepally, V., Moe, N.B.: A decade of agile methodologies: Towards explaining agile software development. Journal of Systems and Software 85(6) (2012) 1213-1221

6. Abrahamsson, P., Warsta, J., Siponen, M., Ronkainen, J.: New directions on agile methods: a comparative analysis. In: Proceedings of the International Conference on Software Engineering. (2003) 244-254

7. Holmström Olsson, H., Alahyari, H., Bosch, J.: Climbing the "stairway to heaven". In: Proceeding of the Euromicro Conference on Software Engineering and Advanced Applications, Cesme, Izmir, Turkey (2012)

8. Kerievsky, J.: Industrial XP: Making XP work in large organizations. Executive Report Vol. 6, No. 2, Cutter Consortium (2005)

9. McMahon, P.: Extending agile methods: A distributed project and organizational improvement perspective. In: Systems and Software Technology Conference. (2005)

10. Lagerberg, L., Skude, T., Emanuelsson, P., Sandahl, K., Stahl, D.: The impact of agile principles and practices on large-scale software development projects: A multiple-case study of two projects at ericsson. In: ACM/IEEE International Symposium on Empirical Software Engineering and Measurement, Baltimore, MD, USA (2013) 348-356

11. Albuquerque, C., Antonino, P., Nakagawa, E.: An investigation into agile methods in embedded systems development. In: Computational Science and Its Applications. Volume 7335 of Lecture Notes in Computer Science. Springer (2012) 576-591

12. Shen, M., Yang, W., Rong, G., Shao, D.: Applying agile methods to embedded software development: A systematic review. In: Proceedings of the International Workshop on Software Engineering for Embedded Systems, IEEE (2012) 30-36

13. Müller, M., Sazama, F., Debou, C., Dudzic, P., Abowd, P.: Survey - State of Practice "Agile in Automotive". Technical report, KUGLER MAAG CIE GmbH (2014)

14. Kaisti, M., Mujunen, T., Mäkilä, T., Rantala, V., Lehtonen, T.: Agile principles in the embedded system development. In: Agile Processes in Software Engineering and Extreme Programming. Volume 179 of Lecture Notes in Business Information Processing., Rome, Italy, Springer (2014) 16-31

15. Shull, F., Singer, J., Sjøberg, D.I.K., eds.: Guide to Advanced Empirical Software Engineering. Springer London, London (2008)

16. Goodman, L.A.: Snowball Sampling. The Annals of Mathematical Statistics 32(1) (1961) 148-170

17. Fisher, R.A.: On the Interpretation of $\chi^{2}$ from Contingency Tables, and the Calculation of P. Journal of the Royal Statistical Society 85(1) (January 1922) 87

18. van Manen, H., van Vliet, H.: Organization-wide agile expansion requires an organization-wide agile mindset. In: Product-Focused Software Process Improvement. Lecture Notes in Computer Science, Helsinki, Finland, Springer (2014) 48-62

19. Petersen, K., Wohlin, C.: A comparison of issues and advantages in agile and incremental development between state of the art and an industrial case. Journal of Systems and Software 82(9) (2009) 1479-1490

20. van Waardenburg, G., van Vliet, H.: When agile meets the enterprise. Information and Software Technology 55(12) (2013) 2154-2171

21. Ambler, S.W., Lines, M.: Disciplined Agile Delivery. 1 edn. IBM Press (2012) 